\title{
Evidence for Two Distinct Effector-Binding Sites in Threonine Deaminase by Site-Directed Mutagenesis, Kinetic, and Binding Experiments ${ }^{\dagger}$
}

\author{
Peter M. Wessel, Emmanuelle Graciet, Roland Douce, and Renaud Dumas* \\ UMR 1932, Laboratoire Mixte CNRS/INRA/Aventis, 14-20 Rue Pierre Baizet, 69263 Lyon, France
}

Received July 12, 2000; Revised Manuscript Received September 27, 2000

\begin{abstract}
A three-dimensional structure comparison between the dimeric regulatory serine-binding domain of Escherichia coli D-3-phosphoglycerate dehydrogenase [Schuller, D. J., Grant, G. A., and Banaszak, L. J. (1995) Nat. Struct. Biol. 2, 69-76] and the regulatory domain of E. coli threonine deaminase [Gallagher, D. T., Gilliland, G. L., Xiao, G., Zondlo, J., Fisher, K. E., Chinchilla, D., and Eisenstein, E. (1998) Structure $6,465-475]$ led us to make the hypothesis that threonine deaminase could have two binding sites per monomer. To test this hypothesis about the corresponding plant enzyme, site-directed mutagenesis was carried out on the recombinant Arabidopsis thaliana threonine deaminase. Kinetic and binding experiments demonstrated for the first time that each regulatory domain of the monomers of A. thaliana threonine deaminase possesses two different effector-binding sites constituted in part by Y449 and Y543. Our results demonstrate that Y449 belongs to a high-affinity binding site whose interaction with a first isoleucine induces conformational modifications yielding a conformer displaying a higher activity and with enhanced ability to bind a second isoleucine on a lower-affinity binding site containing Y543. Isoleucine interaction with this latter binding site is responsible for conformational modifications leading to final inhibition of the enzyme. Y449 interacts with both regulators, isoleucine and valine. However, interaction of valine with the high-affinity binding site induces different conformational modifications leading to reversal of isoleucine binding and reversal of inhibition.
\end{abstract}

Biosynthetic threonine deaminase (EC 4.2.1.16; TD) ${ }^{1}$ is a pyridoxal phosphate enzyme that catalyzes the deamination of threonine into $\alpha$-ketobutyrate.

Allosteric regulation of bacterial TD by its effectors isoleucine and valine was studied initially in the pioneer works of Umbarger (1) and Monod et al. (2). The kinetics of the reaction were further investigated extensively using an overproduced recombinant Escherichia coli enzyme (39). E. coli TD exhibits sigmoidal substrate saturation behavior in the absence of isoleucine. The feedback inhibitor isoleucine decreases the affinity for threonine and increases the cooperativity of the kinetics. On the other hand, valine increases the affinity for threonine, decreases the degree of cooperativity of the kinetics, and reverses the inhibition by isoleucine.

The kinetics of the reaction catalyzed by TD have been studied for various species, including bacteria $(10-12)$, yeast (13), and plants (14-16). The effect of isoleucine and valine on these enzymes was shown to be similar to that described for the E. coli enzyme. However, some differences exist at

This work is dedicated to the memory of Peter M. Wessel. We thank Dr. Ken Palett and Dr. Dave Cole from Aventis Crop Sciences for their contribution to the grant of Peter's Ph.D. Peter was also supported by a training grant (BIO-CT98-5074) from The European Community.

* Corresponding author. E-mail: renaud.dumas@aventis.com. Telephone: 334728522 96. Fax: 33472852297.

${ }^{1}$ Abbreviations: BSA, bovine serum albumin; DTT, dithiothreitol; IPTG, isopropyl $\beta$-D-thiogalactoside; PGD, D-3-phosphoglycerate dehydrogenase; PLP, pyridoxal 5'-phosphate; TD, threonine deaminase; WT, wild-type. the level of enzyme kinetics and the allosteric effect of isoleucine. Indeed, in the absence of isoleucine, the yeast (13) enzyme exhibits sigmoidal kinetics, similar to those of the E. coli enzyme, whereas the Salmonella typhimurium (11), Bacillus subtilis (10), and Spinacia oleracea enzymes (15) show Michaelis-Menten behavior. Furthermore, the yeast enzyme appears to be different from the other enzymes in view of its activation by low concentrations of isoleucine (13).

$\mathrm{TD}$, like many pyridoxal phosphate enzymes, has been shown to be stimulated by monovalent cations. The increase in enzyme activity is particularly strong for plant TD, as demonstrated for the spinach (15) or Paul's scarlet rose enzymes (14).

The bacterial enzyme is a homotetramer with a molecular mass of $224 \mathrm{kDa}$. The resolution of the structure of the $E$. coli enzyme crystallized without its effectors (17) clearly demonstrated that each of the identical subunits is organized into two different domains, in agreement with genetic studies $(4,18)$ (Figure 1A). The N-terminal domain containing the pyridoxal phosphate (PLP) cofactor was proposed to contain the active site (Figure 1A). The C-terminal domain corresponding to the regulatory domain is composed of two homologous subdomains with 2-fold symmetry (Figure 1B). As described in Figure 1A, the $\mathrm{N}$ - and C-terminal domains constitute two globular domains connected by a helix and there are no contacts between the domains within each subunit (17). The tetramer of E. coli TD is a "dimer of dimers" because only weak interactions, involving contacts between catalytic domain, occur between both "dimers" (17). 
$\mathbf{A}$
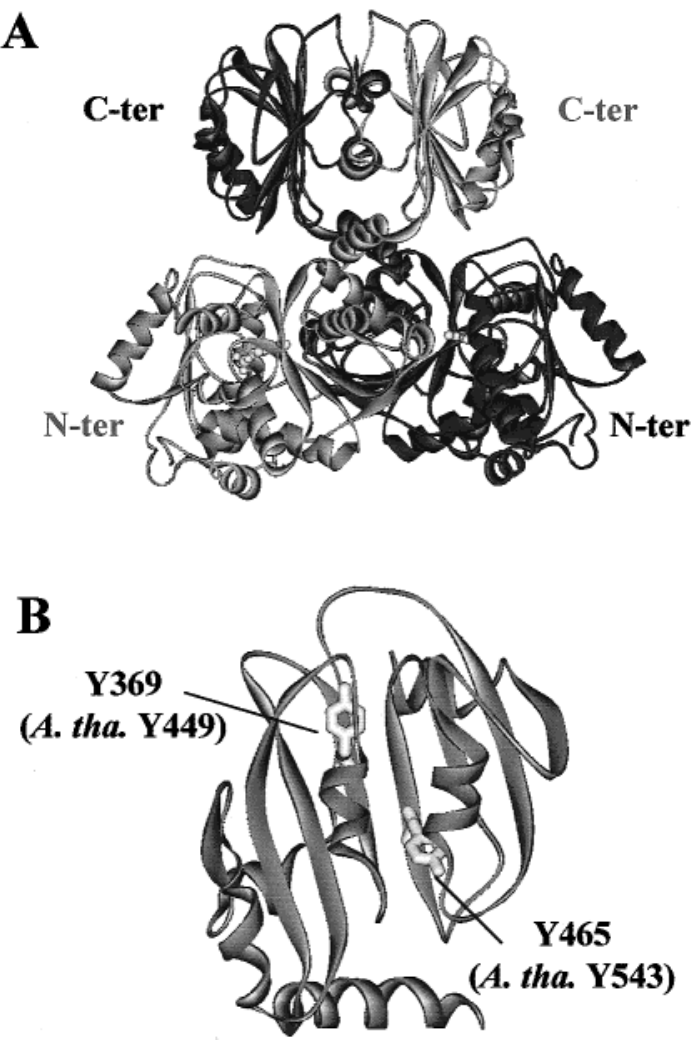

Figure 1: Model of the E. coli TD (from ref 17). (A) The figure shows a TD dimer ribbon model constituted by two equivalent chains in dark and light gray. The $\mathrm{N}$-terminal domain ( $\mathrm{N}$-ter) containing the PLP cofactor (in CPK) was assigned as the active site. The $\mathrm{N}$ - and $\mathrm{C}$-terminal (C-ter) domains constitute two different domains connected by a helix (17). (B) Regulatory domain of the TD subunit. The residues of $E$. coli TD involved in the two effectorbinding sites were selected by structural comparison with PGD (19), amino acid sequence comparison between known TD, and modeling of the A. thaliana enzyme. Among these residues, we investigated the importance of two symmetrical tyrosines (Y449 and Y543) of the A. thaliana enzyme which correspond to Y369 and Y465 (in sticks) of the TD E. coli enzyme, respectively.

Thus, most of the interactions involved in the quaternary structure take place between the regulatory domain of each "dimer" (17).

A three-dimensional structure comparison between TD and the allosteric enzyme D-3-phosphoglycerate dehydrogenase (PGD) (19) disclosed that the regulatory domains of both enzymes have similar folds (17). Indeed, two regulatory domains of PGD associate with 2-fold symmetry to form a dimeric regulatory domain which bears strong similarities with the TD regulatory domain (Figure 1B) (17). Since PGD has been cocrystallized with its regulatory effector serine (19), and each dimeric regulatory domain interface of PGD contains two symmetrical serine-binding sites, it is attractive to make the hypothesis that TD could also possess two effector-binding sites per regulatory domain. Additional three-dimensional structure comparisons between E. coli TD and PGD allowed us to determine several putative residues of $E$. coli TD corresponding to those involved in the effectorbinding site of PGD. From these residues, only those conserved among all the known TD amino acid sequences were selected. At last, modeling of the Arabidopsis thaliana regulatory domain using the Internet Swiss-Model server (20) allowed us to determine that the orientation of the selected residues is similar between the E. coli and A. thaliana TD. These preliminary observations led us to make the hypothesis that F352 and Y369 would be involved in one binding site and that E442 and Y465 participate in a second binding site of the E. coli TD (Figure 1B). In this paper, the importance of these two tyrosines in the effector-binding site is investigated in the A. thaliana enzyme.

To test the hypothesis that TD possesses two binding sites per regulatory domain, we have overproduced and purified the A. thaliana enzyme. Then, we have designed and overproduced in $E$. coli two mutants of the enzyme in which the two tyrosines, corresponding in A. thaliana to Y449 and Y543, are modified. The wild-type and mutant enzymes were further characterized by steady-state kinetics and equilibrium binding experiments.

\section{EXPERIMENTAL PROCEDURES}

Materials. Restriction endonucleases were supplied by New England Biolabs. Isopropyl $\beta$-D-thiogalactoside (IPTG) was supplied by Boehringer (Meylan, France), and amino acids were supplied by Sigma-Aldrich (Steinheim, Germany). Oligonucleotides used for PCR amplification and sitedirected mutagenesis were obtained from Oligo Express (Paris, France). Radioactive $\left[{ }^{3} \mathrm{H}\right]$ isoleucine and $\left[{ }^{3} \mathrm{H}\right]$ valine were purchased from Amersham Pharmacia Biotech.

Strains. E. coli strain DH5 $\alpha$ was used for cloning and mutagenesis, while E. coli strain BL21pLysS was used for recombinant protein overproduction.

Construction of the pET30TD Plasmid Encoding Mature A. thaliana TD. The pET30TD plasmid encoding mature $A$. thaliana threonine deaminase was constructed by PCR using an A. thaliana (Wasilenski) cDNA bank with a proofreading PWO polymerase. The oligonucleotide corresponding to the coding sequence (21) was designed to have a NdeI restriction site with insertion of an ATG codon instead of the codon corresponding to the first amino acid (threonine 46) of the putative mature $A$. thaliana sequence. The oligonucleotide corresponding to the complementary sequence was constructed to insert a SalI restriction site after the TGA stop codon. The PCR product was digested with NdeI and SalI and cloned into a pET30a+ plasmid digested with NdeI and SalI to give the pET30TD vector. Sequencing of the entire insert was carried out, and the sequence was in complete agreement with the previously described A. thaliana TD sequence (21).

Site-Directed Mutagenesis. Site-directed mutagenesis were carried out with the pET30TD vector and using the QuickChange Site-Directed Mutagenesis Kit (Stratagene). Oligonucleotides were designed with replacement of tyrosine 449 or 543 with leucine and for modification of the restriction enzyme profile for identification of mutants. Complete sequencing of the mutants was also performed and showed no mutations other than the desired ones.

Expression and Purification of the Wild-Type and Mutant Enzymes. Transformed E. coli BL21pLysS cells were grown at $28{ }^{\circ} \mathrm{C}$ in $750 \mathrm{~mL}$ of $2 \mathrm{YT}$ medium containing $100 \mathrm{mM}$ $\mathrm{KH}_{2} / \mathrm{K}_{2} \mathrm{HPO}_{4}(\mathrm{pH} 7.5), 100 \mathrm{mM} \mathrm{KCl}$, and the appropriate antibiotics $(34 \mu \mathrm{g} / \mathrm{mL}$ chloramphenicol and $50 \mu \mathrm{g} / \mathrm{mL}$ kanamycin). IPTG was added to a final concentration of 0.5 $\mathrm{mM}$ when bacterial growth was equivalent to an $A_{600}$ of 0.8 . Cells were further grown for $15 \mathrm{~h}$ at $28{ }^{\circ} \mathrm{C}$ and harvested by 
centrifugation at $2000 \mathrm{~g}$ for $15 \mathrm{~min}$. The pellet was resuspended in buffer A [50 mM Hepes $(\mathrm{pH} 7.5)$ and $1 \mathrm{mM}$ EDTA] containing protease inhibitors (5 $\mathrm{mM} \gamma$-aminocaproic acid and $1 \mathrm{mM}$ benzamidine), $1 \mathrm{mM}$ dithiothreitol (DTT), and isoleucine. Wild-type and mutant TD were purified with the same protocol except that isoleucine required for protein stabilization was used in all purification steps at a concentration of $1 \mathrm{mM}$ for the wild-type enzyme and $5 \mathrm{mM}$ for mutant enzymes. The cells were sonicated for $20 \mathrm{~min}$ at $0{ }^{\circ} \mathrm{C}$ on a Vibra-cell disruptor. Following centrifugation at $28000 \mathrm{~g}$ for 30 min, the pellet was discarded and the supernatant was applied to a Fractogel DEAE EMD 650 column $(2.6 \mathrm{~cm} \times$ $30 \mathrm{~cm}$ ) (Sigma) equilibrated in buffer A. Elution was performed with a $800 \mathrm{~mL}$ gradient of 0 to $400 \mathrm{mM} \mathrm{KCl}$ in buffer A containing isoleucine. Chromatographic fractions containing threonine deaminase activity were eluted at 300 $\mathrm{mM} \mathrm{KCl}$. These fractions were concentrated on a Macrosep $30 \mathrm{~K}$ concentrator (Filtron) and applied to a HiLoad 26/60 Superdex 200 column (Pharmacia Biotech) previously equilibrated with buffer A containing isoleucine. After elution, threonine deaminase activity was concentrated on a Macrosep 30K concentrator and then applied to a HiLoad 16/10 Q-Sepharose column (Pharmacia Biotech) equilibrated in buffer A. Elution was performed with a $400 \mathrm{~mL}$ gradient of 0 to $400 \mathrm{mM} \mathrm{KCl}$ in buffer A containing isoleucine. Threonine deaminase activity was eluted at $200 \mathrm{mM} \mathrm{KCl}$. The enzyme was further concentrated on a Microsep 30K concentrator (Filtron) to a concentration of $30 \mathrm{mg} / \mathrm{mL}$ and stored at $-80{ }^{\circ} \mathrm{C}$ for several months without any loss of activity.

Electrophoresis and Protein Quantitation. SDS-PAGE was performed according to the method of Chua (22). The protein was quantitated either by the method of Bradford (23) (for crude extracts only) with bovine $\gamma$-globulin as a standard or by measuring $A_{205}$ as described by Scopes (24).

Protein-PLP Stoichiometry. Knowing the amino acid composition of the A. thaliana TD, we have carried out accurate determination of protein concentration and proteinPLP stoichiometry using the molar extinction coefficients of tyrosine, tryptophan, and PLP under alkaline conditions $(0.1 \mathrm{M} \mathrm{NaOH})$ as described previously for threonine synthase (25).

In Vitro Assays of TD. Threonine deaminase activity was assayed in $100 \mathrm{mM} \mathrm{KH} / \mathrm{K}_{2} \mathrm{HPO}_{4}(\mathrm{pH} 8.0$ ) in a final volume of $1 \mathrm{~mL}$. Reactions were initiated by adding threonine $(0-$ $40 \mathrm{mM})$ without or with isoleucine $(0-20 \mathrm{mM})$ and valine $(0-20 \mathrm{mM})$. All stock solutions of amino acids were prepared in $100 \mathrm{mM} \mathrm{KH} / \mathrm{K}_{2} \mathrm{HPO}_{4}(\mathrm{pH} 8.0)$. The progress of the reaction was monitored by the increase in absorbance at $230 \mathrm{~nm}$ due to $\alpha$-ketobutyrate. Enzyme activity was expressed as micromoles of $\alpha$-ketobutyrate produced per minute per milligram of protein using an $\epsilon$ of $536 \mathrm{~mol}^{-1}$ $\mathrm{cm}^{-1}$.

Filtration Binding Assay. Experiments were carried out with a method adapted from those described earlier (26) for the binding of $S$-adenosylmethionine to ribonucleotide reductase. For each measurement, $0.6-1 \mathrm{mg}$ of TD was incubated in $10 \mathrm{~mL}$ of $100 \mathrm{mM} \mathrm{KH} / \mathrm{K}_{2} \mathrm{HPO}_{4}$ buffer $(\mathrm{pH}$ 8.0) containing various concentrations of isoleucine or valine $(5-300 \mu \mathrm{M})$ and $2-5 \mu \mathrm{L}$ of radiolabeled effector $(87.0 \mathrm{Ci} /$ mmol of L-[4,5- $\left.{ }^{3} \mathrm{H}\right]$ isoleucine or $23.0 \mathrm{Ci} / \mathrm{mmol}$ of $\mathrm{L}-[3,4-$ $\left.{ }^{3} \mathrm{H}\right]$ valine). Experiments with radioactive isoleucine or valine concentrations of $>300 \mu \mathrm{M}$ were not possible under our experimental conditions since the level of nonspecific binding became too high. After incubation of TD $\left(30 \mathrm{~min}\right.$ at $\left.25^{\circ} \mathrm{C}\right)$ with different effector concentrations, $10 \mathrm{~mL}$ of TD was separated in three parts of $3 \mathrm{~mL}$ each. The remaining volume of $1 \mathrm{~mL}$ was used for determination of the amount of total radioactivity. The three incubation mixtures of $3 \mathrm{~mL}$ were filtered under vacuum $(25 \mathrm{mmHg})$ on VMWP filters with a $0.05 \mu \mathrm{m}$ pore size (Millipore) inserted on a vacuum filtration unit (Hoefer Scientific Instrument). After filtration, filters were dried at room temperature on Benchkote (Whatman) and the amount of radioactivity was determined in a vial using $8 \mathrm{~mL}$ of Ready Protein scintillation liquid. In parallel, measurements of controls using BSA instead of TD were also carried out in triplicate at identical buffer, ligand, and protein concentrations and subtracted from the amount of radioactivity of TD samples. The difference between TD and BSA filters was compared to the amount of total radioactivity determined from the unfiltered reaction assays and used to determine the stoichiometry of effector binding. Typically, at $105 \mu \mathrm{M}$ isoleucine $(1918 \mathrm{dpm} / \mathrm{nmol}$ of isoleucine) and with $5 \mathrm{nmol}$ of wild-type TD (on a monomeric basis; in 3 $\mathrm{mL}$ of binding buffer), a difference of about $18000 \mathrm{dpm}$ can be determined after subtraction of the amount of radioactivity bound to BSA filters from that bound to TD filters. Other assays, using threonine synthase [a PLP enzyme purified in our laboratory (25)] as controls, gave the same results as those obtained with BSA. For reversal of isoleucine binding by valine, the protein was first incubated for $5 \mathrm{~min}$ with $100 \mu \mathrm{M}$ isoleucine and radiolabeled isoleucine. Then, various concentrations $(0-15 \mathrm{mM})$ of nonradioactive valine were added, and incubation was continued for $30 \mathrm{~min}$ at 25 ${ }^{\circ} \mathrm{C}$.

Kinetic Data Analyses. Kinetic data were fitted with the appropriate theoretical equations by using the KaleidaGraph program (Abelbeck Software).

Structure Analyses. Stucture analyses of E. coli PGD and TD PDB files were carried out with the WebLab ViewerPro program (Molecular Simulations Inc.). Modeling of the $A$. thaliana sequence was carried out using the Internet SwissModel server (20).

\section{RESULTS}

Overproduction and Purification of Wild-Type and Mutant A. thaliana TD. To obtain a mature protein without its transit peptide, we determined the putative cleavage site of $A$. thaliana TD by homology with the Lycopersicon esculentum enzyme for which the N-terminal amino acid sequence has been reported (27). Expression of a sequence beginning at amino acid 46 (putative cleavage site) yielded production of high amounts of soluble protein. Lengthening the sequence (including the entire peptide transit) or shortening the sequence (beginning at amino acid 71) resulted in production of the insoluble protein. The mutant and wild-type enzymes were purified following a protocol identical to that documented in Figure 2 and described in Experimental Procedures. Typically, a culture of $750 \mathrm{~mL}$ allowed us to obtain $17 \mathrm{mg}$ of pure enzyme.

As Figure 2 shows, the recombinant $A$. thaliana enzyme is composed of $59800 \mathrm{Da}$ subunits. Gel filtration experiments carried out on Superdex 200 in the absence of 


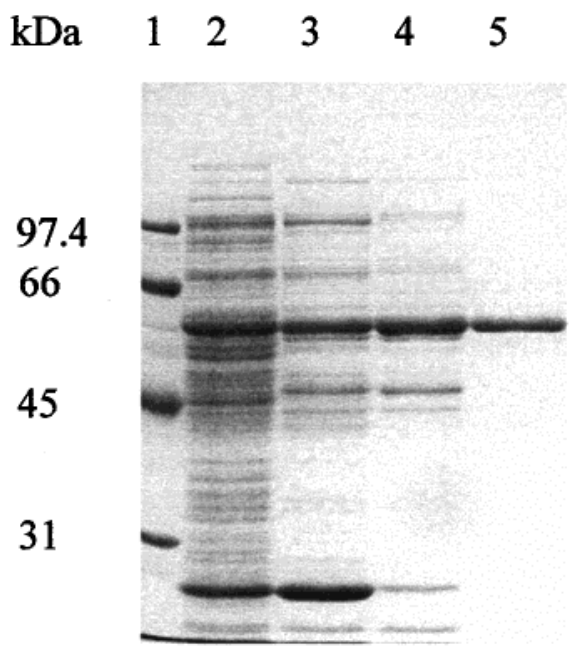

FIGURE 2: Documentation of the purification procedure for the wildtype $A$. thaliana TD by SDS-PAGE. Proteins were separated on a $10 \%$ polyacrylamide $(\mathrm{w} / \mathrm{v})$ slab gel under denaturing conditions and stained with Coomassie Brillant Blue R250: lane 1, $M_{\mathrm{r}}$ markers; lane 2, soluble proteins of the E. coli crude extract (30 $\mu \mathrm{g})$; lane 3, EMD DEAE $650(\mathrm{M})$ pool $(20 \mu \mathrm{g})$; lane 4 , S200 pool $(10 \mu \mathrm{g})$; and lane 5, Q-Sepharose pool $(5 \mu \mathrm{g})$. An identical procedure was used to purify Y449L and Y543L.

isoleucine indicated that the wild-type enzyme behaves as a tetramer under our experimental conditions (not shown). The PLP content determined for the wild-type TD showed a stoichiometry of $1 \mathrm{~mol}$ of PLP per monomer. The recombinant enzyme was strongly activated by monovalent cations such as $\mathrm{K}^{+}, \mathrm{Li}^{+}, \mathrm{NH}_{4}^{+}$, and $\mathrm{Na}^{+}$with an increase of activity of 3.0-, 2.8-, 2.1-, and 1.7-fold, respectively. The activation curves followed hyperbolic behavior, and the $K_{0.5}$ value of activation for the most active monovalent cation $\left(\mathrm{K}^{+}\right)$was determined to be $3.1 \mathrm{mM}$. In contrast, bivalent cations such as $\mathrm{Mg}^{2+}$ or $\mathrm{Ca}^{2+}$ led to inhibition of enzyme activity by a factor of 3.0 or 3.2, respectively. Optimum activity was found at $\mathrm{pH} 8.0$ in $100 \mathrm{mM} \mathrm{KH} \mathrm{H}_{2} / \mathrm{K}_{2} \mathrm{HPO}_{4}$. In this buffer containing a saturating threonine concentration $(40 \mathrm{mM})$ and at $25^{\circ} \mathrm{C}$, the specific activity of the purified wild-type TD was 400

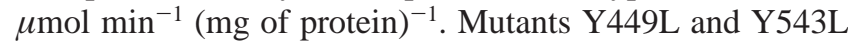
exhibited the same physicochemical and catalytic properties as those determined for the wild-type enzyme with respect to molecular mass, PLP content, cation requirement, optimum $\mathrm{pH}$, and specific activity.

Kinetic Evidence for Two Different Effector-Binding Sites. In the absence of isoleucine, the threonine saturation curve followed Michaelis-Menten behavior for the wild-type and both mutant enzymes. Furthermore, Y449L and Y543L mutants displayed a specific activity [400 $\mu \mathrm{mol} \mathrm{min}^{-1}(\mathrm{mg}$ of protein $)^{-1}$ ] and an apparent $K_{\mathrm{M}}$ for threonine $(7.9 \mathrm{mM})$ nearly identical to those determined for the wild-type enzyme (Figure 3), indicating that mutation of Y449 and Y543 has no effect on the catalytic domain. In the presence of isoleucine, substrate kinetics became sigmoidal and the $K_{0.5}$ value for threonine was increased for the wild-type and mutant enzymes (Figure 3) compared to the $K_{\mathrm{M}}$ value determined in the absence of isoleucine. However, the concentration of isoleucine required for enzyme inhibition was much higher for the two mutants than for the wild-type enzyme (Figures 3 and 4).

Inhibition by isoleucine of Y449L and Y543L mutants was strongly altered compared to that of the wild-type enzyme

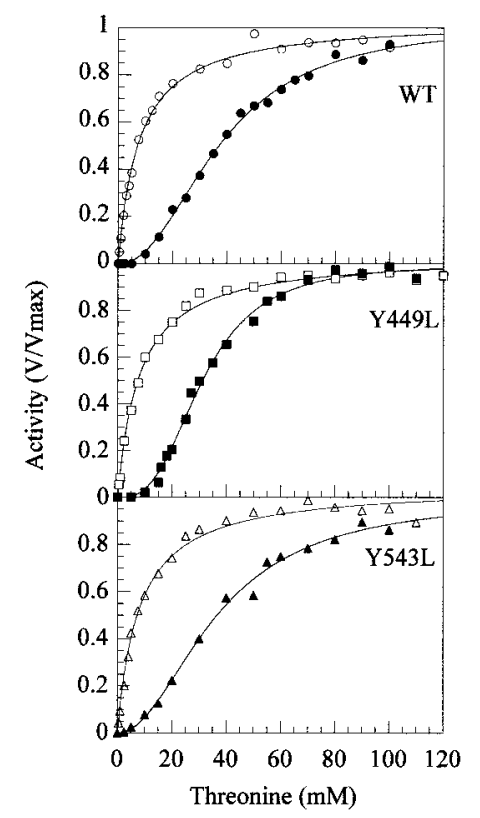

FIGURE 3: Steady-state kinetics of wild-type TD and the Y449L and Y543L mutants. Assays were carried out without effector (open symbols) or with isoleucine (filled symbols). $V_{\max }$ corresponds to $400 \mu \mathrm{mol} \mathrm{min} \mathrm{mg}^{-1} \mathrm{mg}^{-1}$. The isoleucine concentrations used in this experiment are close to the $K_{0.5}$ values for isoleucine determined in Figure 4 (for the WT, $70 \mu \mathrm{M}$; for the Y449L and Y543L mutants, $2.5 \mathrm{mM}$ ). The data corresponding to the assays carried out without effector were fitted by an hyperbolic equation [WT, $K_{\mathrm{M}}$ (threonine) $=7.64 \pm 0.40 \mathrm{mM}$; Y449L, $K_{\mathrm{M}}$ (threonine $)=7.96 \pm 0.42 \mathrm{mM}$; and Y543L, $K_{\mathrm{M}}$ (threonine $)=7.99 \pm 0.56 \mathrm{mM}$ ]. Data corresponding to the assays carried out with isoleucine were fitted by a Hill equation [WT, $K_{0.5}($ threonine $)=37.79 \pm 1.24 \mathrm{mM}$ and $n_{\mathrm{H}}=2.19$ \pm 0.10 ; Y449L, $K_{0.5}$ (threonine) $=31.18 \pm 0.71 \mathrm{mM}$ and $n_{\mathrm{H}}=$ $2.89 \pm 0.15$; and Y543L, $K_{0.5}$ (threonine) $=36.63 \pm 2.45$ and $n_{\mathrm{H}}$ $=2.04 \pm 0.18]$.

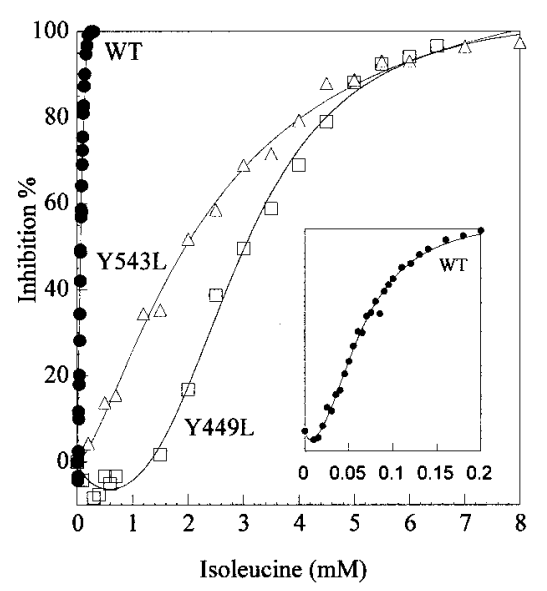

FIGURE 4: Effect of isoleucine on the activity of wild-type TD and the Y449L and Y543L mutants. Reactions were monitored in buffer containing $40 \mathrm{mM}$ threonine. The data corresponding to mutant Y543L were fitted with a Hill equation $\left[K_{0.5}\right.$ (inhibition $)=2.42 \pm$ $0.19 \mathrm{mM}$ and $\left.n_{\mathrm{H}}=1.44 \pm 0.10\right]$. Data corresponding to the wildtype enzyme and mutant Y449L were fitted with a hyperbolic equation followed by a Hill equation as described below: inhibition $\%=\left[\left(-V_{\mathrm{a}} S\right) /\left(K_{0.5 \mathrm{act}}+S\right)\right]+\left[\left(V_{\mathrm{i}} S^{\left.n_{\mathrm{H}}\right)} /\left(K_{0.5 \text { inhib }}+S^{\left.n_{\mathrm{H}}\right)}\right)\right.\right.$, where $V_{\mathrm{a}}$ and $V_{\mathrm{i}}$ are the maximal level of activation and inhibition, respectively, and $S$ is the concentration of isoleucine [WT, $K_{0.5}$ (activation) $=2.81 \pm 4.51 \mu \mathrm{M}, K_{0.5}$ (inhibition) $=64.18 \pm 0.98 \mu \mathrm{M}$, and $n_{\mathrm{H}}=$ $2.19 \pm 0.06$; and mutant Y449L, $K_{0.5}$ (activation) $=0.31 \pm 0.13$ $\mathrm{mM}, K_{0.5}($ inhibition $)=2.94 \pm 0.04 \mathrm{mM}$, and $\left.n_{\mathrm{H}}=3.06 \pm 0.12\right]$.

(Figure 4). Indeed, the concentration of isoleucine needed to reach $50 \%$ inhibition of activity of mutants Y449L and 


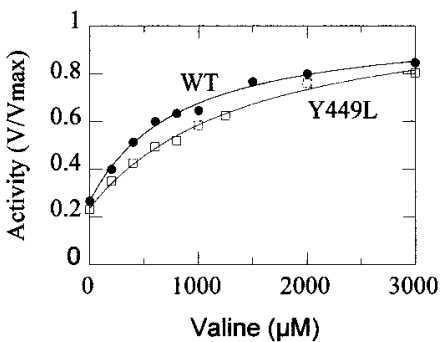

FIGURE 5: Reversal of isoleucine inhibition by valine on wildtype TD and mutant Y449L. Assays were carried out at isoleucine concentrations close to the $K_{0.5}$ values for isoleucine (for the WT enzyme, $70 \mu \mathrm{M}$; and for the mutant Y449L, $3 \mathrm{mM}$ ) (Figure 4). Reactions were monitored in buffer containing $20 \mathrm{mM}$ threonine. $V_{\max }$ values were determined in buffer without isoleucine. The data were fitted with a hyperbolic equation for the WT enzyme $\left[K_{0.5-}\right.$ (valine) $=0.79 \pm 0.08 \mathrm{mM}]$ and for mutant Y449L $\left[K_{0.5}\right.$ (valine) $=1.47 \pm 0.21 \mathrm{mM}]$.

Y543L has been increased by a factor 45 and 38, respectively (Figure 4). As shown in Figure 4 (inset), the plot of the extent of inhibition of the wild-type enzyme as a function of the concentration of isoleucine exhibited two components. A first decreasing curve (activation) defining a site of high affinity for isoleucine was followed by a second increasing curve (inhibition) defining a second site of lower isoleucine affinity. Thus, the wild-type $A$. thaliana TD is activated at low isoleucine concentrations $(0-10 \mu \mathrm{M})$. On the other hand, higher isoleucine concentrations led to inhibition of the wildtype enzyme. This behavior prevented us from fitting the curve with a classical Hill equation. However, we attempted to fit the curve by the sum of a hyperbolic equation and a Hill equation to account for activation and inhibition of enzyme activity, respectively. This sequential equation did not allow us to accurately determine the $K_{0.5}$ value for activation (less than $5 \mu \mathrm{M}$ ) but gave a good estimate of the $K_{0.5}$ for inhibition with a value of $64 \mu \mathrm{M}$ (determined with a saturating threonine concentration of $40 \mathrm{mM}$ ). Interestingly, Y449L and Y543L mutants exhibited different behaviors with respect to isoleucine concentration. As described in Figure 4, the mutant Y449L showed more clearly the phenomenon of activation by isoleucine, but at a higher range of isoleucine concentrations (up to $500 \mu \mathrm{M}$ ) compared to the wild-type enzyme. Isoleucine concentrations of $>1 \mathrm{mM}$ led to inhibition of Y449L. Use of the sequential equation described above allowed us to determine a $K_{0.5}$ values for activation and inhibition of 0.31 and $2.94 \mathrm{mM}$, respectively (values determined with $40 \mathrm{mM}$ threonine) (Figure 4). In contrast, mutant Y543L did not show any activation at low isoleucine concentrations. Moreover, this mutant was inhibited at slightly lower isoleucine concentrations than mutant Y449L, with a $K_{0.5}$ value of $2.42 \mathrm{mM}$ (determined at $40 \mathrm{mM}$ threonine) (Figure 4).

As demonstrated in Figure 5, the inhibition of the wildtype enzyme by isoleucine can be reversed by valine. Reversal of inhibition can be fitted with a hyperbolic equation yielding a $K_{0.5}$ for reactivation of the enzyme by valine of $0.79 \mathrm{mM}$ (in the presence of $70 \mu \mathrm{M}$ isoleucine). Mutants Y449L and Y543L exhibited different behaviors with respect to valine. Indeed, inhibition of mutant Y449L by isoleucine can be reversed by addition of valine $\left(K_{0.5}\right.$ of $1.47 \mathrm{mM}$ for valine for reactivation in the presence of $3 \mathrm{mM}$ isoleucine; see Figure 5), whereas mutant Y543L became insensitive to

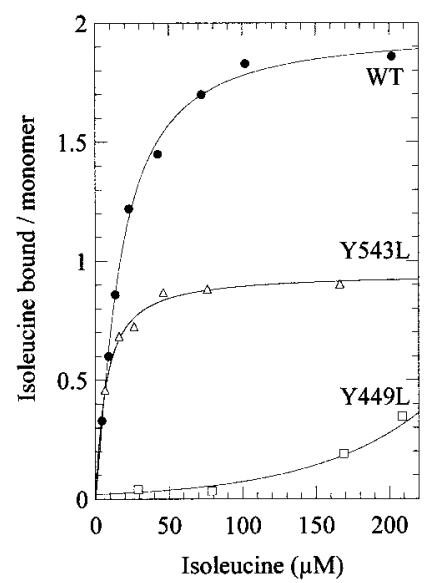

FIGURE 6: Isoleucine binding to wild-type TD and the Y449L and Y543L mutants. Data corresponding to the assays carried out with the wild-type enzyme were fitted by a Hill equation $\left[K_{0.5}\right.$ (isoleucine) $=16.51 \pm 1.06 \mu \mathrm{M}$ and $\left.n_{\mathrm{H}}=1.26 \pm 0.08\right]$. Data corresponding to the assays carried out with Y543L were fitted with a hyperbolic equation $\left[K_{\mathrm{d}}\right.$ (isoleucine) $\left.=7.03 \pm 0.79 \mu \mathrm{M}\right]$.

valine over the range of valine concentrations that were tested $(\leq 3 \mathrm{mM})$.

The different behaviors observed with mutants Y449L and Y543L at the level of isoleucine activation and reversal of isoleucine inhibition by valine strongly indicate that $A$. thaliana TD contains two different effector-binding sites. To determine the effector specificity of both binding sites, we carried out equilibrium binding experiments with the wildtype and mutant enzymes.

Isoleucine Binding to the Wild-Type and Mutant Enzymes. Binding experiments with $\left[{ }^{3} \mathrm{H}\right]$ isoleucine carried out with the wild-type enzyme clearly confirmed the existence of two isoleucine-binding sites per $A$. thaliana TD subunit (Figure 6). Indeed, the best fit of isoleucine binding data with the wild-type enzyme was achieved by using a Hill equation, which allowed us to determine the saturation of two binding sites with a $K_{0.5}$ value of $16.5 \mu \mathrm{M}$ and a slight positive cooperativity $\left(n_{\mathrm{H}}=1.3\right)$. In marked contrast, isoleucine binding to mutants Y449L and Y543L was greatly altered (Figure 6). Indeed, at isoleucine concentrations of $<200 \mu \mathrm{M}$, saturation isotherms showed that mutant Y543L was able to bind $1 \mathrm{~mol}$ of isoleucine per monomer only. In this case, the best fit of the isoleucine binding data was achieved by using a hyperbolic equation yieding a $K_{\mathrm{d}}$ value of $7.0 \mu \mathrm{M}$. Isoleucine binding to mutant $\mathrm{Y} 449 \mathrm{~L}$ was altered differently. As Figure 6 shows, no saturation could be reached in the isoleucine range investigated herein $(\leq 200 \mu \mathrm{M}$ isoleucine), suggesting that mutant $\mathrm{Y} 449 \mathrm{~L}$ remains able to bind isoleucine but with a much lower affinity than the wild-type enzyme and mutant Y543L. In summary, these equilibrium binding experiments demonstrated the existence of two isoleucine binding sites per monomer. Moreover, these results demonstrate that only one of these sites has been altered in mutant Y543L, whereas two sites were affected in mutant Y449L.

Valine Binding to the Wild-Type and Mutant Enzymes. Filtration binding experiments using valine with the wildtype and mutant Y543L enzymes gave similar results, with a saturation at $0.5 \mathrm{~mol}$ of valine per monomer at valine concentrations of $<200 \mu \mathrm{M}$ (Figure 7). In each case, the best fit of the binding data was achieved by using a hyperbolic equation leading to the determination of a $K_{\mathrm{d}}$ 


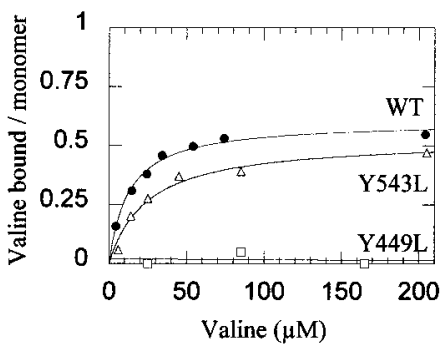

FIGURE 7: Valine binding to wild-type TD and Y543L mutants. Data were fitted with a hyperbolic equation for the wild type $\left[K_{\mathrm{d}}-\right.$ $($ valine $)=12.65 \pm 1.37 \mu \mathrm{M}]$ and mutant Y543L $\left[K_{\mathrm{d}}(\right.$ valine $)=$ $24.47 \pm 5.27 \mu \mathrm{M}]$.

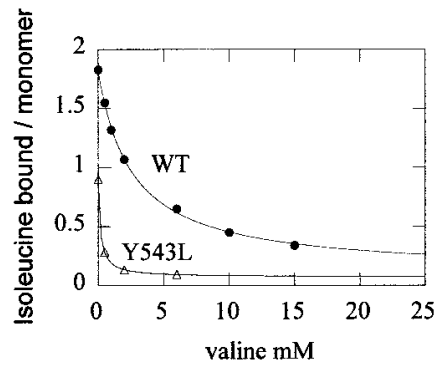

FIGURE 8: Reversal of isoleucine binding to wild-type TD and mutant Y543L by valine. Data were fitted with a hyperbolic equation for the wild-type enzyme $\left[K_{\mathrm{d}}(\right.$ valine $\left.)=2.57 \pm 0.17 \mathrm{mM}\right]$ and for mutant Y543L $\left[K_{\mathrm{d}}(\right.$ valine $\left.)=171.63 \pm 3.94 \mu \mathrm{M}\right]$.

value of 12 and $24 \mu \mathrm{M}$ for the wild-type and mutant Y543L enzymes, respectively. In contrast and as shown in Figure 7, mutant Y449L became unable to bind valine. These results demonstrate that Y543L is able to bind low valine concentrations, whereas Y449L has lost this ability. To understand the effect of valine on the reversal of inhibition and on the binding of isoleucine, we carried out further equilibrium binding experiments to follow reversal of isoleucine binding by valine.

Reversal of Isoleucine Binding by Valine. Reversal of isoleucine binding was investigated with a fixed radioactive isoleucine concentration of $100 \mu \mathrm{M}$ and with increasing concentrations of nonradioactive valine. Displacement of the small amount of isoleucine ( $0.1 \mathrm{~mol}$ per monomer) bound to Y449L was completely achieved with $1 \mathrm{mM}$ valine (not shown). As shown in Figure 8, the reversal of bound isoleucine from the wild-type TD and from mutant Y543L by valine can be fitted with a hyperbolic equation, allowing the determination of $K_{\mathrm{d}}$ values of 2.5 and $0.17 \mathrm{mM}$, respectively. Using this equation, the estimated value for the remaining isoleucine, at saturing valine concentrations, on the wild-type enzyme and Y543L was 0.1 and $0.07 \mathrm{~mol}$ of isoleucine per monomer, respectively, suggesting that valine binding led to almost complete reversal of isoleucine from the two binding sites.

\section{DISCUSSION}

The purified recombinant $A$. thaliana TD exhibits a specific activity of $400 \mu \mathrm{mol} \mathrm{min}{ }^{-1} \mathrm{mg}^{-1}$ (at $25^{\circ} \mathrm{C}$ ), higher than that determined for the recombinant $E$. coli enzyme (210

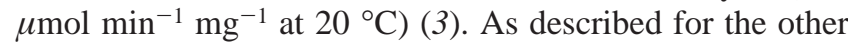
plant enzymes previously studied $(14,15)$, A. thaliana TD has a strong requirement for monovalent cations and in particular for $\mathrm{K}^{+}$which was found to stimulate the enzyme by about 3 -fold. The purified recombinant $A$. thaliana TD obeys Michaelis-Menten kinetics in the absence of isoleucine as described for A. thaliana desalted crude extract (16) and for S. typhimurium (11), B. subtilis (10), and S. oleracea purified enzymes (14). This behavior is different from that established for the E. coli (3) and Saccharomyces cerevisiae (13) enzymes, which exhibit sigmoidal kinetics in the absence of isoleucine.

A three-dimensional structure comparison between the $E$. coli TD (17) and PGD (19) showed that the dimeric regulatory domain of PGD bears strong similarities to the regulatory domain of TD (17). Since the interface of each dimeric regulatory domain of PGD contains two serine binding sites $(19,28,29)$, we can imagine that TD could also possess two effector-binding sites per regulatory domain. A careful three-dimensional structure comparison between E. coli TD and PGD allows us to identify several putative residues of $E$. coli TD corresponding to the effector-binding site of PGD. Among these amino acids, only those conserved among bacterial, fungal, and plant TD amino acid sequences were further selected. These preliminary observations indicated that F352 and Y369 would be involved in one binding site and that E442 and Y465 participate in the second binding site of the E. coli TD (Figure 1). Further observations allow us to determine that the conformation of these four amino acids is similar between the E. coli and A. thaliana TD as shown by the comparison of the structure of the bacterial enzyme and modeling of the A. thaliana enzyme (20; Internet Swiss-Model program, not shown). In this paper, we determined the importance of the corresponding A. thaliana Y449 and Y543 in the mechanism of regulation of plant TD.

Previous studies with double (residues 447 and 451) and triple (residues 447, 451, and 454) mutants were carried out on a helix belonging to one subdomain of the regulatory domain of $E$. coli TD (9). These double and triple mutants are less sensitive to both effectors with decreases in isoleucine affinity of about 15- and 100-fold, respectively (9). However, these mutations have also decreased the affinity for threonine in the absence of isoleucine (9). This observation suggests therefore that these mutations have also induced modification of conformations at the level of the active site leading to enzyme inhibition. In contrast to these experiments, single mutations at Y449 and Y543 of the A. thaliana enzyme led to a strong decrease in isoleucine affinity without altering the kinetics with respect to threonine in the absence of effector.

Kinetic measurements of isoleucine and valine regulation on Y449L and Y543L mutants demonstrate for the first time that $A$. thaliana TD has two structurally different allosteric effector-binding sites per monomer. Indeed, our results clearly show that mutation of either Y449 or Y543 leads to a dramatic decrease in the level of enzyme inhibition by isoleucine since the $K_{0.5}$ values of this effector for both mutants are increased by about 40-fold compared to that of the wild-type enzyme (Figure 4). Furthermore, our results demonstrate that these two sites exhibit different features with respect to valine and isoleucine. Indeed, mutant Y543L is not activated by low concentrations of isoleucine, whereas mutant Y449L is activated by higher isoleucine concentrations than the wild-type enzyme (Figure 4). In addition, isoleucine inhibition of mutant Y449L can be reversed by valine (Figure 5), whereas mutant Y543L became insensitive to valine reversal (not shown). 
Observations of the effect of isoleucine on the wild-type enzyme led to the hypothesis that one of the two binding sites would correspond to a high-affinity binding site for isoleucine (Figure 4, inset). One hypothesis is that isoleucine binding at this high-affinity site would induce a modification of the conformation at the level of the catalytic domain which leads to a conformer exhibiting higher activity. At the same time, isoleucine binding in this high-affinity site would also induce a modification of the conformation at the level of the regulatory domain, allowing the second binding site with lower affinity to interact with a second isoleucine. Finally, the binding of this second isoleucine would lead to a second modification of the conformation in the catalytic domain, leading to the observed inhibition.

Our results indicate that mutation of Y449 altered dramatically the concentration needed for isoleucine activation (Figure 4), suggesting that Y449 would belong to the highaffinity isoleucine-binding site. However, since mutation of Y543 suppresses completely the activation by isoleucine (Figure 4), Y543 could be also involved in this high-affinity isoleucine-binding site. To distinguish between these two hypotheses, filtration binding experiments were further carried out to determine the effector specificity of both binding sites.

Our filtration binding experiments with isoleucine and the wild-type enzyme have demonstrated definitively the binding of $2 \mathrm{~mol}$ of isoleucine per monomer, in agreement with the structural comparison and our kinetic results. Binding experiments with isoleucine and mutants Y449L and Y543L showed that mutations have altered in a different way the binding of isoleucine. Our experiments showed that each monomer of mutant Y543L is able to bind $1 \mathrm{~mol}$ of isoleucine with a $K_{\mathrm{d}}$ similar of those determined for the wildtype enzyme. This observation suggests that the unaffected site of Y543L (Y449) can always binds with high affinity 1 mol of isoleucine. It must be pointed out that the binding of isoleucine to mutant $\mathrm{Y} 543 \mathrm{~L}$ is reached at a concentration 100-fold lower than those required for inhibition (Figures 4 and 6). This latter result suggests, therefore, that binding of isoleucine to Y449 is not responsible for the observed inhibition. Furthermore, this result is in agreement with the hypothesis that Y449 belongs effectively to the high-affinity site for isoleucine. Filtration binding experiments carried out on mutant Y449L indicated that this mutant is always able to bind isoleucine but with a much lower affinity (Figure 6). Once again, this result is in accordance with the hypothesis that Y449 belongs to the high-affinity site for isoleucine since mutation of Y449 increases the isoleucine concentration needed to fill this site and thus the concentration required to induce modification of the conformation leading to the binding of isoleucine to the other site.

Filtration binding experiments carried out with valine had showed that the wild-type enzyme and mutant Y543L bind in a similar way $1 \mathrm{~mol}$ of valine per two monomers, demonstrating that Y449 is also involved in the binding of valine. In agreement with this finding, no binding of valine has been detected for mutant Y449L (Figure 7). Thus, our results show that the high-affinity binding site for isoleucine is also able to interact with valine on the wild-type enzyme. One hypothesis is that valine competes with isoleucine for interaction with Y449. However, binding of valine to Y449 could lead to a conformational modification, different from

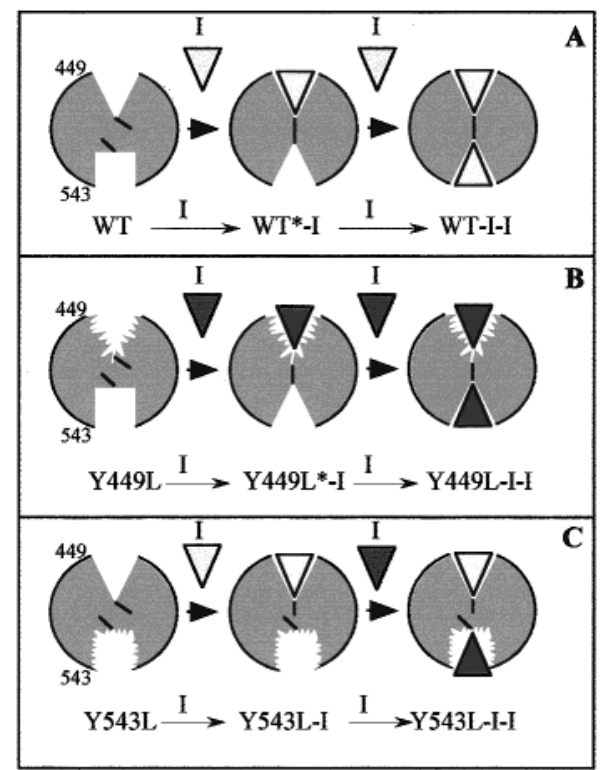

FIGURE 9: Working model to account for the interactions of isoleucine with the two binding sites of each regulatory domain of A. thaliana TD (see the text): (A) wild-type enzyme, (B) mutant Y449L, and (C) mutant Y543L. Open circles correspond to a regulatory domain (see Figure 1). White triangles correspond to low isoleucine concentrations. Dark triangles correspond to high isoleucine concentrations. Asterisks correspond to a conformer exhibiting higher activity upon binding of $1 \mathrm{~mol}$ of isoleucine per monomer. Binding of $2 \mathrm{~mol}$ of isoleucine (I-I) per monomer leads to inhibition of enzyme activity. For mutant Y543L, discontinuous lines between the two regulatory sites indicate that the site corresponding to Y543 has lost its ability to undergo a conformational change upon binding of isoleucine to Y449. The site containing Y449 behaves also as a binding site for valine. The effect of valine is described in the text only.

those induced by isoleucine and preventing the binding of the second isoleucine to Y543. Such a hypothesis is supported by the reversibility of isoleucine binding (Figure 8 ) and reversal of inhibition (Figure 5) upon addition of valine.

Filtration binding experiments with isoleucine and the wild-type enzyme led to the saturation of two binding sites per monomer (Figure 6), whereas experiments carried out with valine allow saturation of only one binding site per dimer (one-half per monomer) (Figure 7). This result is another indication that valine and isoleucine binding leads to different modifications of conformation. Indeed, binding of 2 mol of isoleucine to one monomer does not prevent the binding of an additional $6 \mathrm{~mol}$ of isoleucine to the three other monomers belonging to the tetramer. However, binding of 2 mol of valine to A. thaliana TD would display strong negative cooperativity. In this case, binding of valine to two monomers would induce conformational modifications that prevent the binding of valine to the two other monomers of the tetramer.

In conclusion, the results of kinetic and binding experiments allow us to propose a new working model for the interaction between the effector-binding sites and their effect on the regulation of activity. Our model states that each regulatory domain of each monomer constituting the native tetramer of $A$. thaliana TD possesses two effector-binding sites corresponding to Y449 and Y543 (Figure 9). Our model suggests that Y449 belongs to a high-affinity binding site whose interaction with isoleucine leads to conformational 
modifications corresponding to a conformer displaying a higher activity. This conformation is able to bind more efficiently isoleucine on a second lower-affinity binding site containing Y543. Isoleucine interaction with Y543 is responsible of conformational modifications leading to final inhibition of the enzyme (Figure 9). Y449 of the wild-type enzyme interacts with both regulators, isoleucine and valine. However, interaction of valine with Y449 induces different conformational modifications leading to reversal of isoleucine binding and reversal of inhibition.

Mutant Y543L shows no activation at low isoleucine concentrations (Figure 4), and there is no effect of valine on the reversal of isoleucine inhibition. To explain this behavior, we have to introduce in our model the idea that mutation at Y543 has also altered the transfer of information between the two binding sites. In other words, upon binding of isoleucine to the high-affinity binding site, mutant Y543L has lost its ability to undergo a modification of the conformation leading to a conformer exhibiting a higher activity and a better affinity for the second isoleucine. Thus, in mutant Y543L, the alteration of the communication between Y543 and Y449 would also alter the ability of valine to reverse isoleucine inhibition without affecting the binding of valine on Y449.

Finally, this work demonstrates that the sequential binding of two isoleucines inside each regulatory domain of the $A$. thaliana TD induces modification of the conformation of the catalytic domains leading to inhibition of activity. In addition to this mechanism, our results also indicate that regulation of plant TD activity arises from interactions between subunits as shown by cooperativity at the level of ligand binding and threonine kinetic. Despite the differences observed between the plant and bacterial enzymes, this study does not rule out the mechanism of allosteric regulation established for the E. coli TD (2). However, our paper shows that the mechanism of regulation of the plant TD is different and more complicated than that postulated by previous studies (2) on the bacterial enzyme. At this step, cocrystallization of the plant and bacterial TD with the effectors valine and isoleucine will help in understanding the regulation of these complexes.

\section{ACKNOWLEDGMENT}

We are grateful to Drs. Michel Matringe, Claude Alban, Ron Duggleby, and Dominique Job for helpful discussions.

\section{REFERENCES}

1. Umbarger, H. E. (1956) Science 123, 848.
2. Monod, J., Wyman, J., and Changeux, J.-P. (1965) J. Mol. Biol. 12, 88-118.

3. Einsenstein, E. (1991) J. Biol. Chem. 266, 5801-5807.

4. Fisher, K. E., and Eisenstein, E. (1993) J. Bacteriol. 175, 6605-6613.

5. Einsenstein, E. (1994) J. Biol. Chem. 269, 29416-29422.

6. Einsenstein, E., Yu, H. D., and Schwarz, F. P. (1994) J. Biol. Chem. 269, 29423-29429.

7. Einsenstein, E. (1995) Arch. Biochem. Biophys. 316, 311318 .

8. Einsenstein, E., Yu, H. D., Fisher, K. E., Iacuzio, D. A., Ducote, K. R., and Schwarz, F. P. (1995) Biochemistry 34, 9403-9412.

9. Chinchilla, D., Schwarz, F. P., and Eisenstein, E. (1998) J. Biol. Chem. 273, 23219-23224.

10. Hatfield, G. W., and Umbarger, H. E. (1970) J. Biol. Chem. $245,1742-1747$.

11. Decedue, C. J., Hofler, J. G., and Burns, R. O. (1975) J. Biol. Chem. 250, 1563-1570.

12. Hofler, J. G., and Burns, R. O. (1978) J. Biol. Chem. 253, 1245-1251.

13. Betz, J. L., Hereford, L. M., and Magee, P. T. (1971) Biochemistry 10, 1818-1824.

14. Dougall, D. K. (1970) Phytochemistry 9, 959-964.

15. Sharma, R. K., and Mazunder, R. (1970) J. Biol. Chem. 245, 3008-3014.

16. Mourad, G., and King, J. (1995) Plant Physiol. 107, 43-52.

17. Gallagher, D. T., Gilliland, G. L., Xiao, G., Zondlo, J., Fisher, K. E., Chinchilla, D., and Eisenstein, E. (1998) Structure 6, 465-475.

18. Taillon, B. E., Little, R., and Lawther, R. P. (1988) Gene 63, $245-252$.

19. Schuller, D. J., Grant, G. A., and Banaszak, L. J. (1995) Nat. Struct. Biol. 2, 69-76.

20. Peitsch, M. C. (1996) Biochem. Soc. Trans. 24, 274-279.

21. Mourad, G., Emerick, R., Marion, A., and Smith, A. (1998) Plant Physiol. 118, 1534.

22. Chua, N. H. (1980) Methods Enzymol. 69, 434-436.

23. Bradford, M. M. (1976) Anal. Biochem. 72, 248-254.

24. Scopes, R. K. (1974) Anal. Biochem. 59, 277-282.

25. Curien, G., Job, D., Douce, R., and Dumas, R. (1998) Biochemistry 37, 13212-13221.

26. Ollagnier, S., Mulliez, E., Schmidt, P. P., Eliasson, R., Gaillard, J., Deronzier, C., Bergman, T., Gräslund, A., Reichard, P., and Fontecave, M. (1997) J. Biol. Chem. 272, 24216-24223.

27. Samach, A., Hareven, D., Gutfinger, T., Ken-Dror, S., and Lifschitz, E. (1991) Proc. Natl. Acad. Sci. U.S.A. 88, 26782682.

28. Grant, G. A., Schyuller, D. J., and Banaszak, L. J. (1996) Protein Sci. 5, 34-41.

29. Grant, G. A., Xu, X. L., and Hu, Z. (1999) Protein Sci. 8, $2501-2505$.

BI001625C 